\title{
Autonomous Space Shuttle
}

\author{
Jeffrey A. Siders \\ United Space Alliance, LLC \\ Houston, TX 77058, USA \\ tel: 281-282-5658/ fax: 281-282-5810 \\ jeff.a.siders@usa-spaceops.com
}

\author{
Robert H. Smith \\ United Space Alliance, LLC \\ Houston, TX 77058, USA \\ tel: 281-282-5659/ fax: 281-282-5810 \\ robert.h.smith@usa-spaceops.com
}

\begin{abstract}
The continued assembly and operation of the International Space Station (ISS) is the cornerstone within NASA's overall Strategic Plan. As indicated in NASA's Integrated Space Transportation Plan (ISTP), the International Space Station requires Shuttle to fly through at least the middle of the next decade to complete assembly of the Station, provide crew transport, and to provide heavy lift up and down mass capability. The ISTP reflects a tight coupling among the Station, Shuttle, and OSP programs to support our Nation's space goals.
\end{abstract}

While the Shuttle is a critical component of this ISTP, there is a new emphasis for the need to achieve greater efficiency and safety in transporting crews to and from the Space Station. This need is being addressed through the Orbital Space Plane (OSP) Program. However, the OSP is being designed to "complement" the Shuttle as the primary means for crew transfer, and will not replace all the Shuttle's capabilities.

The unique heavy lift capabilities of the Space Shuttle is essential for both ISS, as well as other potential missions extending beyond low Earth orbit. One concept under discussion to better fulfill this role of a heavy lift carrier, is the transformation of the Shuttle to an "un-piloted" autonomous system. This concept would eliminate the loss of crew risk, while providing a substantial increase in payload to orbit capability.

Using the guidelines reflected in the NASA ISTP, the autonomous Shuttle a simplified concept of operations can be described as; "a re-supply of cargo to the ISS through the use of an un-piloted Shuttle vehicle from launch through landing". Although this is the primary mission profile, the other major consideration in developing an autonomous Shuttle is maintaining a crew transportation capability to ISS as an assured human access to space capability.
Although the current Shuttle has the capability in numerous areas to operate without crew interaction, there are still many tasks that can only be accomplished through a direct action by a crewmember. In addition to the standard or nominal crew actions required during a Shuttle mission, the availability of the crew to detect or react to off-nominal or contingency situations is an essential function that would be very difficult to replace.

The primary set of changes required to move to an autonomous Shuttle are those associated with the replacement of the standard or "nominal" crew operations. This replacement can be accomplished either through onboard automation or creating the ability for ground or ISS commandable operations. In addition, the challenges and issues associated with retaining situational awareness and dealing with failures or contingencies are much more complex and difficult to resolve.

When addressing the transformation to an un-piloted Shuttle system, numerous hardware, software and procedural changes will be required to both flight and ground systems. An important consideration in the incorporation of these changes is the need to perform these modifications as a "block" update to the Shuttle system, to minimize the impacts and complexities associated with the operations of a mixed fleet.

Converting the Shuttle fleet to an autonomous system will be challenging and expensive. Although an autonomous Shuttle eliminates the risk for loss of crew, the risk to mission success could potentially be increased. Previous Shuttle experience in space has demonstrated that the human presence provides an invaluable capability to successfully react to any situation that may arise. This capability cannot be easily "automated" or replaced.

\footnotetext{
${ }^{1}$ All of the authors are employed by United Space Alliance. The views expressed in this paper are solely those of the authors and do not necessarily represent an official position or view of the authors' employer.
}

\footnotetext{
Copyrigh_ 2003 by United Space Alliance, LLC. Published by the IEEE Conference with permission. These materials are sponsored by the National Aeronautics and Space Administration under Contract NAS9-20000. The U.S. Government retains a paid-up, nonexclusive, irrevocable worldwide license in such materials to reproduce, prepare derivative works, distribute copies to the public, and perform publicly and display publicly, by or on behalf of the U.S. Government. All other rights are reserved by the copyright owner. Paper \#1177
} 


\section{TABLE OF CONTENTS}

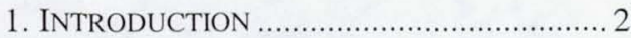

2. CHALLENGES AND ISSUES ......................... 2

3. DEFINITION AND ASSUMPTIONS ................ 3

4. CONCEPT OF OPERATIONS......................... 4

5. CHANGES AND MODIFICATIONS …............. 5

6. CONCLUSIONS ............................................. 7

7. REFERENCES ........................................... 7

8. BIOGRAPHY ……….............................. 7

\section{INTRODUCTION}

The continued assembly and operation of the International Space Station is the cornerstone within NASA's overall Strategic Plan. As indicated in the Integrated Space Transportation Plan (ISTP), represented by Figure 1, the International Space Station requires Shuttle to fly through at least the middle of the next decade to complete assembly of the Station, provide crew transport, and to provide heavy lift up and down mass capability. The ISTP reflects a tight coupling among the Station, Shuttle, and OSP programs to support our Nation's space goals.

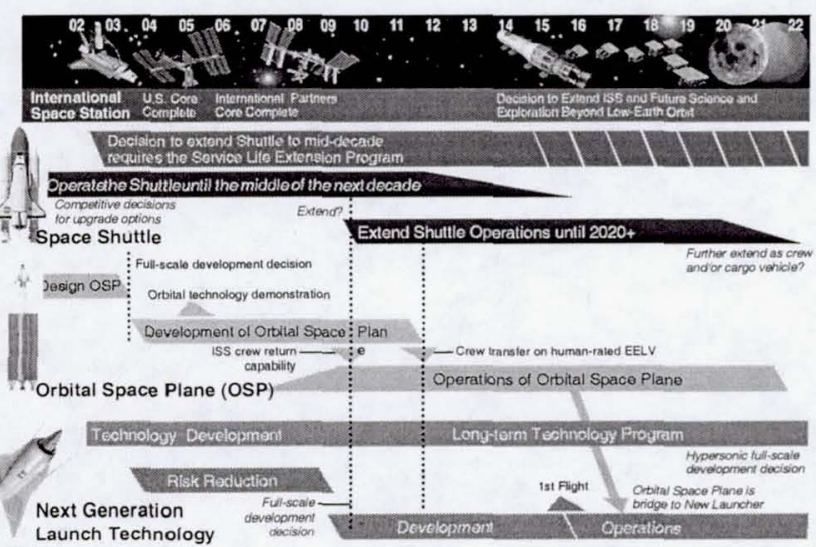

Figure 1: NASA's Integrated Space Transportation Plan

While the Shuttle is a critical component of this ISTP, there is a new emphasis for the need to achieve greater efficiency and safety in transporting crews to and from the Space Station. This need is being addressed through the Orbital Space Plane (OSP) Program. However, the OSP is being designed to "complement" the Shuttle as the primary means for crew transfer, and will not replace the Shuttle.

The unique heavy lift capabilities of the Space Shuttle is essential for both ISS, as well as other potential missions extending beyond low Earth orbit. One concept under discussion to better fulfill this role of a heavy lift carrier, is the transformation of the Shuttle to an "unmanned" autonomous system. This concept would eliminate the loss of crew risk, while providing a substantial increase in payload to orbit capability.

Almost since the awarding of the original Shuttle contracts, the Aerospace community has been proposing ways to improve the Space Shuttle. Among the various improvements proposed was the unmanned Shuttle concept. The first significant unmanned study was undertaken in 1973. Since that time there have been numerous studies pertaining to the Unmanned Orbiter concept. These previous studies were typically slanted towards a particular objective and had limited applicability to current autonomous Shuttle thinking. They focused on feasibility assessments and emphasized automation of nominal procedures with less detail regarding off nominal and higher levels of redundancy. A summary of these studies can be found in the reference [2], and in the following figure.

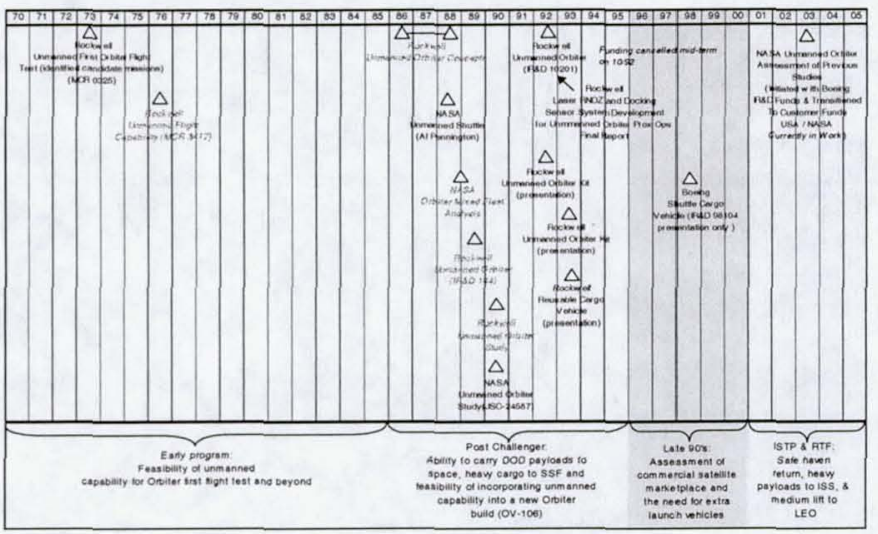

Figure 2: Significant Unmanned Orbiter Studies Produced from 1973 through 2003

This paper addresses the challenges, issues, potential modifications and impacts associated with a transition to an autonomous Shuttle.

\section{Challenges and Issues}

Although an autonomous Shuttle eliminates the risk for loss of crew, it may actually increase the risk for loss of the vehicle during time critical operations, due to the loss of crew situational awareness and intervention for unaccounted problems and malfunctions. Increasing levels of autonomous operations, fault isolation and reconfiguration capabilities would help to reduce this risk.

When addressing the transformation to an un-manned Shuttle system, numerous hardware, software and procedural changes will be required. The primary set of required changes are those associated with the replacement of the 
standard or "nominal" crew operations. This replacement can be accomplished either through onboard automation or creating the ability for ground or ISS commandable operations.

In addition, the challenges and issues associated with maintaining situational awareness and dealing with failures or contingencies are much more complex and difficult to implement in an autonomous system.

Some of the key challenges and issues that would need to be addressed in an autonomous Shuttle include:

\section{$\underline{\text { Ascent }}$}

- Automation of intact and contingency aborts

- Feasibility of taking an autonomous vehicle to TAL/ECAL locations

- Pre-launch Preparation and checkout

- Range safety

- Post insertion configuration

\section{Orbit, Rendezvous and Docking}

- Enhanced Communication System and Coverage

- Situational awareness during nominal and offnominal scenarios

- Contingency operations that previously required EVA, (Ku-jettison, payload bay door closure, etc)

- Automated Rendezvous and Proximity Operations (i.e. redundany, sensors, reflectors, etc)

- Docking and Hatch operations

- Navigation for relative attitudes during the final docking phase (range within 30 feet)

- Avionics automation of the current ODS system

- Potential to utilize other docking mechanisms and concepts such as berthing

- Undocking and separation from ISS

- Breakout capability initiated from ISS, ground and onboard sequences required

Deorbit, Entry and Landing

- Deorbit configuration and burn execution

- APU start

- Overflight of population centers

- Air Data probe deploy

- Autoland

- Landing gear arm/deploy

- Drag chute arm/deploy

- Auto braking \& steering

- Redundant nosewheel steering

- Enhanced ground landing aids

\section{$\underline{\text { General }}$}

- Flight software

$$
\text { - OI release impacts }
$$

- Mission manager to initiate auto sequences based on events

- Hardware automation

- Modification kit design

- Switch emulation

- redundant communications (command and telemetry),

- KSC ground processing, vehicle modification schedule, turnaround planning

- Mission support modifications (ground equipment, simulators, software test facility modifications, Flight Controller training, etc.)

- Mixed Fleet implications, impacts and mitigation

- Payload definition and deployment operations concepts

- Implementation cost and schedule estimates

\section{DEFINITION AND ASSUMPTIONS}

Although the current Shuttle has the capability to operate "autonomously" in numerous areas, there are still many tasks that can only be accomplished through a direct action by a crewmember. Implementation of an autonomous Shuttle would require development of new functional capabilities as well as automation of existing control techniques.

The concept of an autonomous Space Shuttle can be simply defined as the automated replacement of those functions or actions that are typically performed by the crew in today's Shuttle operations. This automation would be accomplished through software and hardware modifications to emulate crew actions, (such as switch throws or mechanism deployments), and modifications to the flight and ground systems to allow for Shuttle commanding via ground or Space Station personnel. Various degrees of autonomy would also be required depending on the time criticality of the function being replaced. They are defined as;

- Fully Autonomous - Time critical functions that must be performed automatically at a specific time, independent of ground control, to achieve mission success and avoid damage or loss of the vehicle. (Aborts)

- Semi-Autonomous - Time critical functions with multiple windows of opportunity completed automatically with external ground command initiation to achieve mission success and preserve vehicle integrity.

- Manual - manual operations that are accomplished by external ground control commands performed at a convenient time with no performance penalty due to delay. 
An important consideration is the need to perform these modifications without impact to planned Shuttle flights, to minimize the impacts to the on-going Space Station missions, and the complexities associated with the operations of a mixed fleet.

The other major consideration in developing an autonomous Shuttle is maintaining a crew transportation capability to ISS as a backup to the OSP to provide an assured human access to space capability. This would involve making the Shuttle "switchable", meaning that any modifications would not be of the magnitude to preclude being replaced during a normal processing flow. This would maintain the capability to revert back to a crewed Shuttle, if required.

Other guidelines and assumptions associated with the development of an autonomous Shuttle, are summarized below:

\section{Maintaining the Configuration And Capabilities of The Current Shuttle System -}

This assumption is intended to minimize structural or Outer Mold Line (OML) modifications to the Orbiter to maintain the current vehicle certifications. It also implies the current Shuttle Element "architecture" will be maintained, which consists of; an Orbiter vehicle, 2 Solid Rocket Boosters (SRBs), an External Tank (ET), and 3 Space Shuttle Main Engines (SSMEs). (i.e. no LFBB or SSME's on ET, would be considered). The autonomous Orbiter vehicle configuration and supporting elements shall utilize developed / proven Shuttle hardware, software, ground facilities and operational procedures to the fullest extent practicable. Orbiter weight and CG will remain within current certified envelope and structural modifications to the vehicle will be minimized.

\section{ISS Logistic Flights Only}

Autonomous Shuttle would primarily be utilized to re-supply and return payloads from ISS, and will not need to address any other complex mission scenarios, (no automated payload retrieval / deploy, ISS assembly operations, etc). Maintaining a pressurized crew compartment to allow for ISS stowage and RMS access, will be required to support this assumption. In addition, the ISS crew will be required to open and close all interface hatches.

\section{Limited Exposure to Populated Areas}

Un-manned vehicle over-flights of highly populated areas, might raise safety concerns with the range and FAA. For this reason, landings will probably be planned to occur at Edwards (EDW), or some other remote site to minimize how much of the continental US the unmanned vehicle will fly over.

\section{Reduction of Crew Support Equipment}

With the removal of the crew from the Shuttle system, a significant amount of hardware and equipment could be eliminated, to provide in-creased volume and payload weight availability to ISS. Some of these items include:

- Removal of crew seats and consumables (food, clothing, FDF, etc)

- No EVA suits, equipment or tools

- No exercise equipment

- No crew escape or survival equipment

- No LiOH

- No Galley or Waste Control System (WCS)

However, it is also assumed that any modifications would not be of the magnitude to preclude being replaced during a normal processing flow. This would maintain the capability to revert back to a crewed Shuttle, if required.

\section{Crew Preparation and Associated Ground Support Activities}

With an Autonomous Shuttle, no mission specific crew training or planning would be required, (No CAPCOM, FAO, Flight Surgeon, SMS, STA, T-38s, NBL, etc). The elimination of crew training and planning activities would also allow for other pre-mission efficiencies to be realized.

In addition, with an autonomous Shuttle there would be no need for windows, thus eliminating the extensive ground processing refurbishment and replacement activities. No interior lighting or other crew related equipment would be needed during un-docked periods, which would reduce the overall vehicle power requirements. Also, no BFS or SM GPC would be required for ascent or entry. MCC will command all item entries and OPS transitions via a DEU equivalent. Good communications are required for GPC reconfiguration.

\section{CONCEPT OF OPERATIONS}

The autonomous Shuttle would primarily be utilized to resupply and return payloads from ISS, and would not be utilized for any other complex mission scenarios, such as; automated payload retrieval / deploy, Hubble Space Telescope refurbishment, or ISS assembly operations. Maintaining a pressurized crew compartment would be desirable to allow for ISS stowage and RMS access. 
Crew functions would need to be replaced and desired levels of redundancy maintained with either uplinked commands from the ground or ISS and/or onboard software sequences. The ground versus onboard command philosophy would be based on concepts used by other NASA complex spacecrafts (such as HST, GRO, etc.).

\section{Autonomous Ascent Overview}

All pre-launch activities previously performed by the crew, will now be performed by ground personnel. During ascent, the current Shuttle system does not require very much direct interaction from the crew for any routine or standard action, (there is only one nominal switch throw during ascent). Yet the crew is essential during this phase by monitoring all systems and making preparations for an abort, if necessary. The autonomous Shuttle ascent profile will primarily be the same as with the current Shuttle system, with an enhanced focus on maintaining good communications to support any ground commanding that may be required. Abort capabilities for an autonomous Shuttle will be limited.

\section{Autonomous On-Orbit Operations Overview}

Once established on-orbit, (payload bay doors open, vehicle systems operational and communications established), the autonomous Shuttle has to perform the normal crew activities associated with rendezvous and docking to the ISS. Although the major rendezvous maneuvers can be commanded from the ground, much of the final approach and docking will have to be monitored and controlled by the ISS crew. The ISS crew will be controlling the Shuttle in + Rbar attitude with Shuttle nose aft.

Once docking is completed, the ISS crew will open the Shuttle hatch to gain access. Following mated operations, the ISS crew will again monitor and control the undocking and separation maneuvers from ISS.

\section{Autonomous Deorbit and Entry Overview}

The current Shuttle entry and landing mission profile requires direct crew action in several key areas. All of which will need to be replaced in an autonomous entry. Although a nominal ISS resupply mission would allow the ISS crew access to configure the Shuttle for deorbit and entry, considerations must be made for a mission profile that does not achieve an ISS docking for whatever reason. In this case, the autonomous Shuttle must have the capability to be configured for entry through automated or ground commanding. Some of these typical activities include:

- Thermal conditioning

- Flight Control System checkout

- APU's started prior to entry
- Payload bay door closure

- Deorbit targeting, preparation, configuration and execution

Following the deorbit burn, the nominal entry profile for the autonomous Shuttle is the same as the current Shuttle. The guidance and flight control systems are in control. Entry reconfiguration commanding capability will be required to react to any encountered entry dynamics (winds, shears, etc). As the vehicle approaches the landing site, the automated system performs the activities previously executed by the crew, such as; deploying the air data probes and lowering of the landing gear.

The autoland phase of the current Shuttle entry has been worked in the past to a high level of confidence. Autoland guidance was designed to fly consistent with how an actual crew would fly the vehicle. Incorporation of this capability into an autonomous Shuttle would be essential. To successfully accomplish an automated entry and landing a highly accurate navigation system will also be required, (i.e TACAN, MSBLS, GPS, etc).

Although not required, the autonomous Shuttle system might consider incorporation of certain landing optimization capabilities to ensure success. Some of these include;

- Nominal/Close-in Aimpoint selection for touchdown energy control

- Shortfield Speedbrake selection

- Nose Wheel Steering (NWS) activation

- Drag chute deploy and jettison

- Braking

Following landing, the autonomous Shuttle will then be required to perform all vehicle and payload safing operations.

\section{Changes and Modifications}

The transformation to an autonomous Shuttle fleet will require both hardware and software changes, to both flight and ground systems. Incorporation of these modifications will need to be performed as a "block" update, to minimize the impacts and complexities associated with the operations of a mixed fleet. Modifications for autonomous capability must also be implemented without impacting the Shuttle manifest support to ISS. Some of the major modifications include;

\section{Automation of crew switches -}

Primary emphasis would be placed of the nominal mission profile switch throws. Additional switch automation for 
contingency or abort scenarios would be highly desirable. This modification also includes a significant impact to the Shuttle Flight Software system to also support the additional ground or ISS commanding capability. Some of these key switch throws and other crew actions that need to be automated include:

- 1 ascent switch throw (ADI to LVLH)

- On-orbit configuration (PLBD, ECLSS, etc)

- Star tracker / IMU alignment

- OMS and RCS switch configuration

- Deorbit Preparation and execution

- 2 Switches associated with Air Data Probe deploy

- 6 Switches associated with APU "START/RUN"

- 2 Switches associated with Landing Gear "ARM" and deploy (DN)

- Post landing vehicle and payload safing

Additional Instrumentation and IVHM -

Additional instrumentation to replace the crew situational awareness will be necessary for ground insight of the Shuttle systems. An IVHM system would be required for an autonomous Shuttle to be able to observe, detect and react to onboard or external situations. This modification would take the form of vehicle sensors, cameras, and data management systems.

\section{Enhanced Shuttle Communications System -}

Today, the crew is utilized to configure the communications hardware for the communications system in order to gain the second s-band link for failure scenarios taking command on the primary link. Without the crew, one failure can take out communications to the autonomous vehicle. This makes the autonomous vehicle zero fault tolerant in command and telemetry without adding additional redundancy. Previous studies recommended different solutions to solve this problem such as redundant S-Band FM or UHF. For these designs, redundancy is obtained through ground stations. Redundancy through TDRS S-Band would be preferred and requires further analysis and design.

In order to meet the needs of an autonomous Shuttle system as previously defined, the onboard vehicle communications system will most likely need to be upgraded, (i.e. Ka-band Phased Array Antenna (PAA) system). This would allow communications with the ground or ISS, without requiring opening of the payload bay doors and deployment of the current Ku-Band antenna.

\section{Ground Communications System -}

In addition to the modifications to the ground systems required by the "Enhanced Shuttle Communications System" modification described above, additional ground communication modifications would be required. These modifications would take the form of additional ground communication stations, upgraded systems, etc.

\section{Docking Adapter and Hatch Modifications -}

The ISS crew will required to open and close the Shuttle hatch during the mated operations. To accomplish this, the current Shuttle hatch will need to be redesigned to allow operation from external to the vehicle.

\section{Rendezvous and Proximity Operations Aids -}

To aid in the automated rendezvous and docking, modifications will be required in the areas of; enhanced camera system, additional ISS reflectors, enhanced radar system and ISS monitoring and commanding system.

\section{Entry and Landing Optimization (Optional)}

Without a crew to optimize the landing performance, additional modifications can be incorporated to provide additional margins for success. These include:

- Nose Wheel Steering (NWS) activation

- Drag chute deploy and jettison

- Braking

\section{Additional Landing Aid Equipment}

Incorporation of GPS or redundant MSBLS, calibrated to achieve the autoland accuracy requirements. (Autoland requires accuracy of $0.1 \mathrm{deg}$ alignment error vs the current accuracy requirement of $0.15 \mathrm{deg}$ alignment error).

\section{Autonomous Shuttle Operations -}

All Shuttle operational processes and procedures will need to be reviewed and updated as required to support autonomous vehicle operations. This includes;

- Flight planning

- Flight Rules and Launch Commit Criteria (LCC) updates.

- Ground processing

- Ground controller training

- Mixed fleet operational impacts

\section{Flight Software -}

Moving to an autonomous Shuttle system will require significant flight software changes. Some of the areas that could be affected include; 
- Flight Control

- Guidance

- Sequencing

- Autoland

- Switches

- CAU Displays

- Auto Pryo landing gear deploy

- Uplinks

- Differential Braking

- Drag Chute

The software impacts in some of these areas may be significant, and could be comparable in size to a normal block update (OI). Early releases of minimum capability may be achieved, possibly with I-load changes and/or patches. Ground applications, ground testing and GSE will be significantly impacted as well.

\section{Conclusions}

Implementation of an autonomous Shuttle system could be pursued as a phased approach of increasing autonomous capability, with the potential for certification during manned missions, as shown in Figure 3. The path towards an ISTP autonomous Shuttle cargo vehicle could begin with nearterm benefits to the current Shuttle system in the areas of safe haven return of the vehicle, and reduced crew workload or crew size.

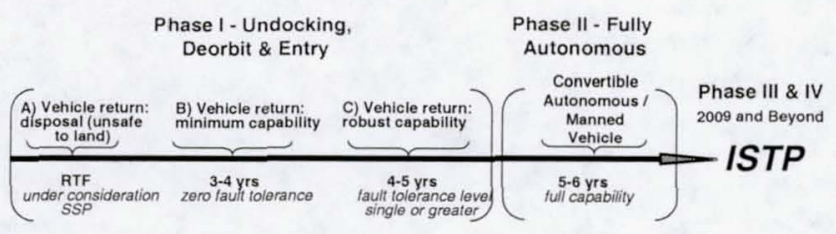

Figure 3: Phased Implementation Approach

The unique heavy lift capabilities of the Space Shuttle is essential for both ISS, as well as other potential missions extending beyond low Earth orbit. The transformation of the Shuttle to an "un-piloted" autonomous system would eliminate the loss of crew risk, while providing a substantial increase in payload to orbit capability.

In addition to ISS cargo support, an autonomous Shuttle can also provide operational flexibility to the current Shuttle operations, in the areas of:

- Returning a damaged Shuttle from the Space Station (Safe Haven)

- Landing the Shuttle should the crew become incapacitated

- Reducing crew workload during normal or complex activities, such as rendezvous/Docking, etc.

- Complementing crew escape implementation by allowing smaller Shuttle crew size (2-4)

- Providing a growth path for a Shuttle derived heavy lift capability (Shuttle-C)

- Providing Assured Access to Station through retrofit to a crewed capability when needed

With the removal of the crew from the Shuttle system, a significant amount of hardware and equipment could also be eliminated, to provide increased volume and payload availability to ISS, (i.e. Removal of crew seats, EVA suits and tools, exercise equipment, crew galley, Waste Control System (WCS) and various fluids and consumables). In addition, the elimination of crew training and planning activities would also allow provide for some pre-mission preparation efficiencies. However, an increase in ground controller training and flight software reconfiguration would probably keep any potential cost savings to a minimum.

Converting the Shuttle fleet to an autonomous system will be challenging and expensive. Projected implementation cost estimates for an autonomous Shuttle system would be in the low $\$$ billions, depending on the degree of modification and system redundancy required. Although an autonomous Shuttle eliminates the risk for loss of crew, the risk to a loss of vehicle or mission success could potentially be increased. Previous Shuttle experience in space has demonstrated that the human presence provides an invaluable capability to successfully react to any situation that may arise. This important capability cannot be easily "automated" or replaced.

\section{REFERENCES}

[1] "Autoland Implementation for Unmanned Orbiter Deorbit/Return Capability, Preliminary Assessment", (April 7, 2003), Rafael de la Torre, Kristina Houston, Chris Lessmann, Sam Cristol

[2] "Autonomous Shuttle Previous Studies Assessment", (November 2003), Jeff Siders, Debra Bailey

\section{BIOGRAPHY}

